\title{
Study on Intelligent Monitoring Technology for Composting of Agricultural and Livestock Wastes
}

\author{
Hualong $\mathrm{Li}^{1,2}$, Miao $\mathrm{Li}^{1(\bowtie)}$, Xuanjiang Yang ${ }^{1,2}$, Zelin $\mathrm{Hu}^{1}$, \\ Zhirun $\mathrm{Ma}^{1}$, and Xianwang $\mathrm{Liu}^{3}$ \\ ${ }^{1}$ Institute of Intelligent Machines, Chinese Academy of Sciences, Hefei, Anhui 230031, \\ China \\ ${ }^{2}$ University of Science and Technology of China, Hefei, Anhui 230026, China \\ ${ }^{3}$ Anhui Agricultural University, Hefei, Anhui 230026, China \\ lihualong2007@163.com, mli@iim.ac.cn
}

\begin{abstract}
Composting is a harmless treatment process of agricultural and livestock wastes are widely used for grass-roots livestock farms. Composting temperature control is the key technical condition in composting process. In most current production process, the mechanical artificial temperature measuring devices were adopted to measure the composting temperature, which could not monitor the composting process resulting in the dumping delay. Meanwhile, the pathogenic microorganisms in compost could not be inactivated effectively. To solve this problem, an intelligent monitoring device was designed to realize the on-line monitoring of the whole process of composting. Users can query the compost temperature in real time by smart phone so as to dump in time to improve the inactivation efficiency of pathogenic microorganisms and reduce the loss of compost nutrients. The composting monitoring experiments showed that the system could monitor the composting process on-line and will have broad application in the field of livestock waste recycling.
\end{abstract}

Keywords: intelligent monitoring; agricultural and livestock waste; harmless treatment; composting temperature

\section{Introduction}

Aerobic composting is the process of absorption, oxidation and decomposition of organic compounds and pathogenic microorganisms in wastes such as livestock, sludge and straw with oxygen and bacteria. In the whole process, the composting temperature directly affect the inactivation of pathogenic microorganisms and the decomposition of organic compounds. Therefore, the composting temperature control is the key technical issue of the composting process [1,2]. In most current production process, the mechanical artificial temperature measuring devices were adopted to

$\bowtie$ Corresponding author. 
measure the composting temperature, which could not monitor the composting process in real time, and would result in the dumping delayed, so that the pathogenic microorganisms in compost could not be inactivated effectively $[3,4]$.

To solve this problem, an intelligent monitoring device was designed to realize the real-time monitoring of the whole process of composting with the temperature sensor, which had the advantages of high temperature measurement accuracy, simple operation. Compared with the traditional use of mechanical temperature measuring instrument, the data support for scientific composting can be provided. Users could query the temperature of the compost online by web page or smartphone APP, and then dump in time, which could save the cost of composting and reduce the loss of compost nutrients.

\section{The design of monitoring systems}

As Figure 1, the system consisted of three parts: data acquisition terminal, server and client. The data acquisition terminal included a data collector and the composting temperature sensor. The sensor was connected to the data collector by RS-485 bus. STM32F103VB (ARM 32-bit Cortex-M3 CPU) was used as the core processor in the data collector, which could achieve the local storage and remote transmission of the data. The server was developed based on the Web platform, SQL Server 2008 and ASP.NET. And it was used to receive data storage and processing. The clients contained two parts: Web query and Android mobile client [5].

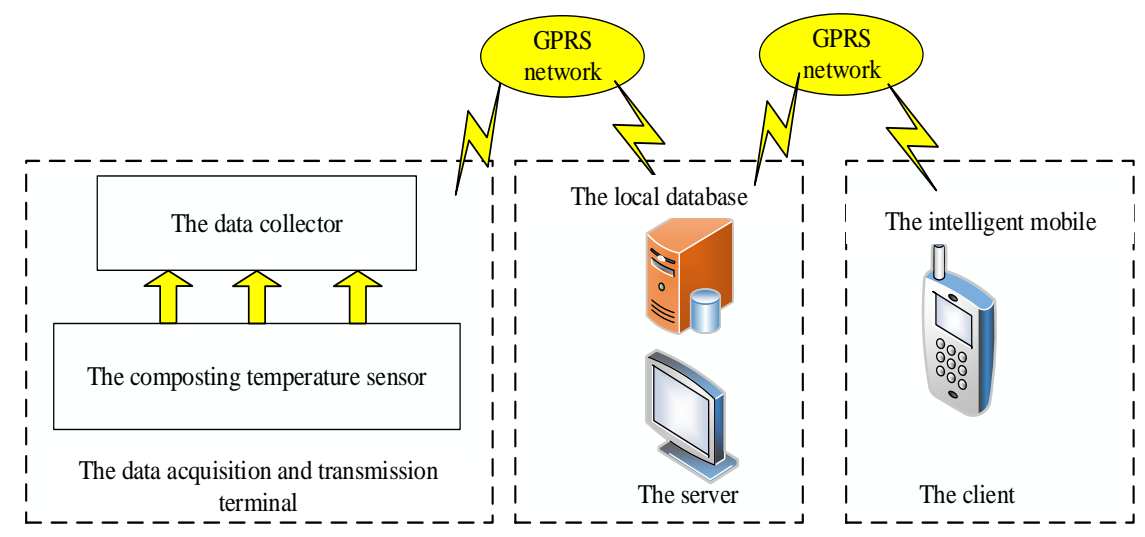

Fig. 1. The diagram of intelligent compost monitoring device 


\subsection{The hardware design}

The data collector adopted the low power consumption microprocessor STM32F103VBT6 as CPU. The system function modules included the RS-485 data acquisition, GPRS wireless data sending, real-time clock, SD card data storage, human-computer interaction and power management (Figure 2).

The RS-485 data acquisition module was used to connect with the composting temperature sensor (CG-01, Hefei heinford Electronic Technology Co. Ltd. The measurement range of air temperature: $-40{ }^{\circ} \mathrm{C} \sim+85{ }^{\circ} \mathrm{C}$, with the measurement accuracy: $\pm 0.3{ }^{\circ} \mathrm{C}$ ). The GPRS wireless data sending module could accomplish the real-time transmission of the composting temperature data to the remote server. Combining with SD card data storage module and the real-time clock module, the temperature data was stored in SD card. This design could effectively solve the data loss which due to the instability of signal transmission and improve the reliability of the real-time monitoring [6].

Human-computer interaction module included a 4.3-inch LCD and a keyboard. The composting temperature could be displayed in real-time on the LCD. The LCD power could be turn off or open automatically, which can reduce system power consumption and extend the LED working life. The keyboard was used for setting of the periodic sampling, filtering and the controlling mode of data transmission. Power management module included a DC12V 48AH large capacity cell group and the corresponding power conversion circuit.

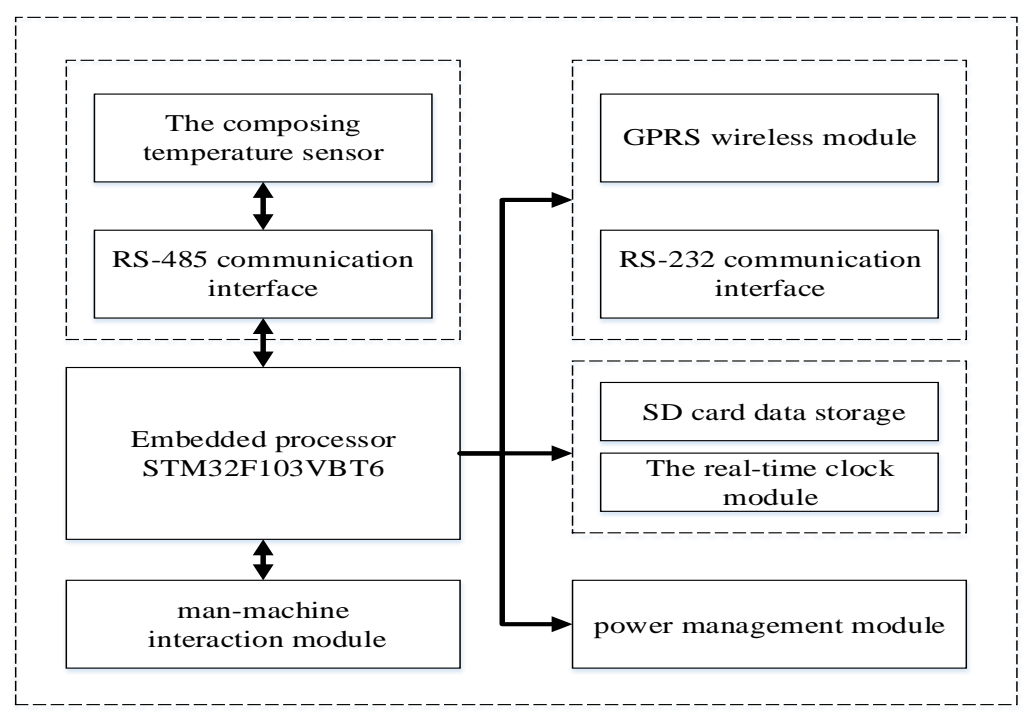

Fig. 2. The data structure diagram of the data collector 


\subsection{The software design}

Headings. The software design of data collector. The monitoring terminal software was written in $\mathrm{C}$ language mainly to complete the collection of environment data and send them to the GPRS network by using Keil integrated development environment as software programming and debugging platform (Figure 3 ).

When the system started, the data collector first determined whether the acquisition time reached, and sent the data acquisition command to the composting temperature sensor. If not, it continued to wait. Then the data collector received the composting temperature data from the sensor after three milliseconds delay, and performed CRC check. If the check result was correct, the data would be sent to the GPRS network. Otherwise the data acquisition instruction would be sent again.

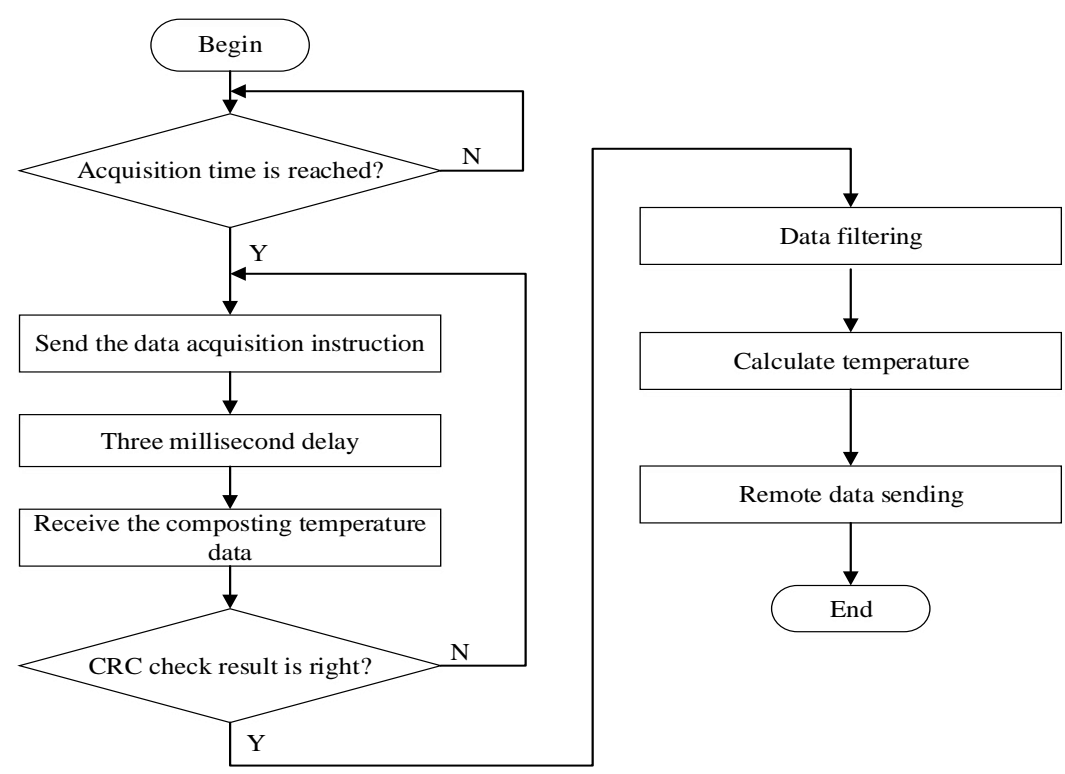

Fig. 3. The data structure diagram of the data collector

To improve the reliability of the sensor measurement and reduce the measurement error caused by interference, the average filtering algorithm of anti-impulse interference was used to process the received temperature data. The average filtering method had the advantages of simple calculation, less time consumption and less storage, which was very suitable for embedded system. 
Headings. The software design of monitoring system. The software of monitoring system platform included three parts: the server-side monitoring centre software, visual system software and the data query android APP.

The server-side monitoring centre software program was developed in Visual Studio 2010 with $\mathrm{C}$ \# programming, which ran on the backend of the server. The service monitoring program established a communication connection with GPRS-DTU unit to accomplish two-way communication between the embedded terminal and the server. After the connection was established, the service monitor would parse the package data sent by the embedded device and store the parsed data in the SQL Server 2008 database. The specific process of the service monitoring centre software was shown in Figure $4[7,8]$.

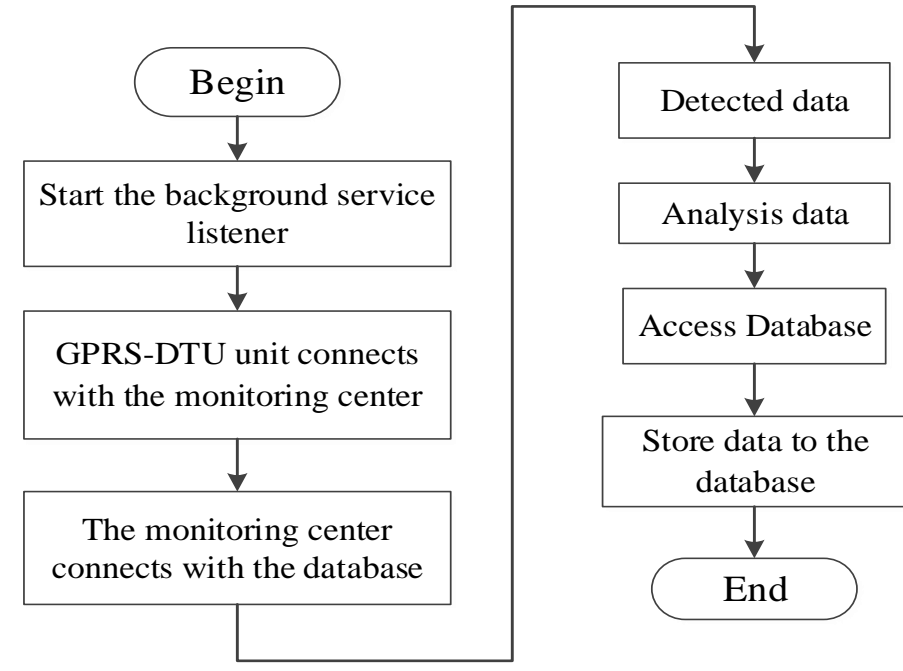

Fig. 4. The software design of data monitoring center

Data visualization platform mainly accomplished the function of data query, data download, data graphical display and remote control of monitoring device. The design process was shown in Figure 5 and the compost temperature monitoring platform web page was shown in Figure 6. 


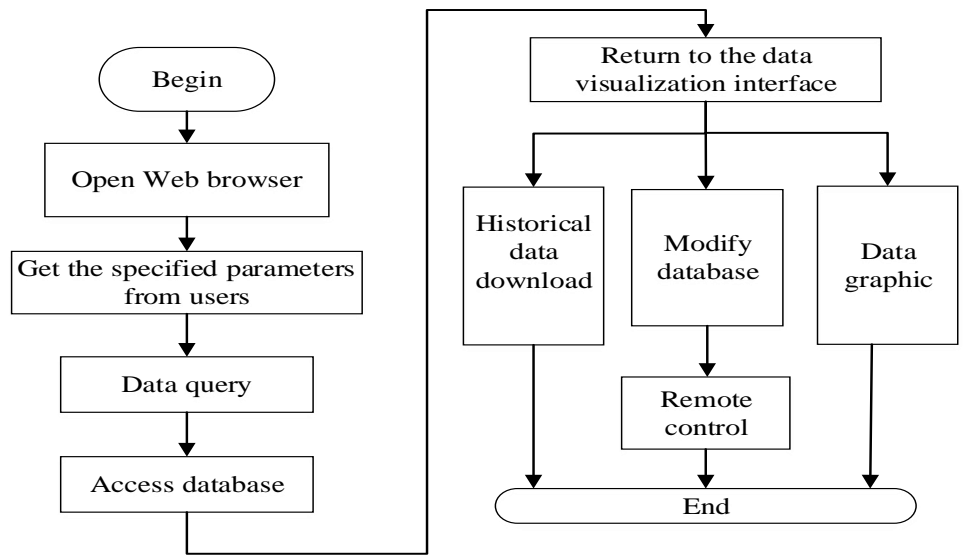

Fig. 5. The software design process of visual system

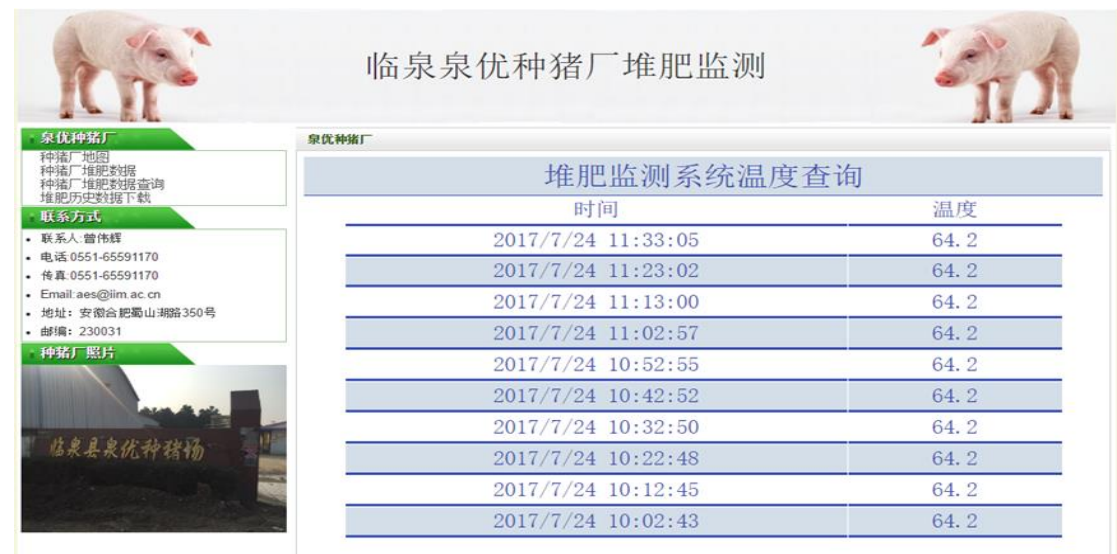

Fig. 6. The compost temperature monitoring platform web page

The data query android APP was developed based on eclipse. The android SDK plug-in was installed in eclipse. With android widget, Android clients could be quickly developed. In addition, the web service program was written on the server side to open the corresponding service port, so that the users could access the database from the Android client. The design process was shown in Figure 7. 


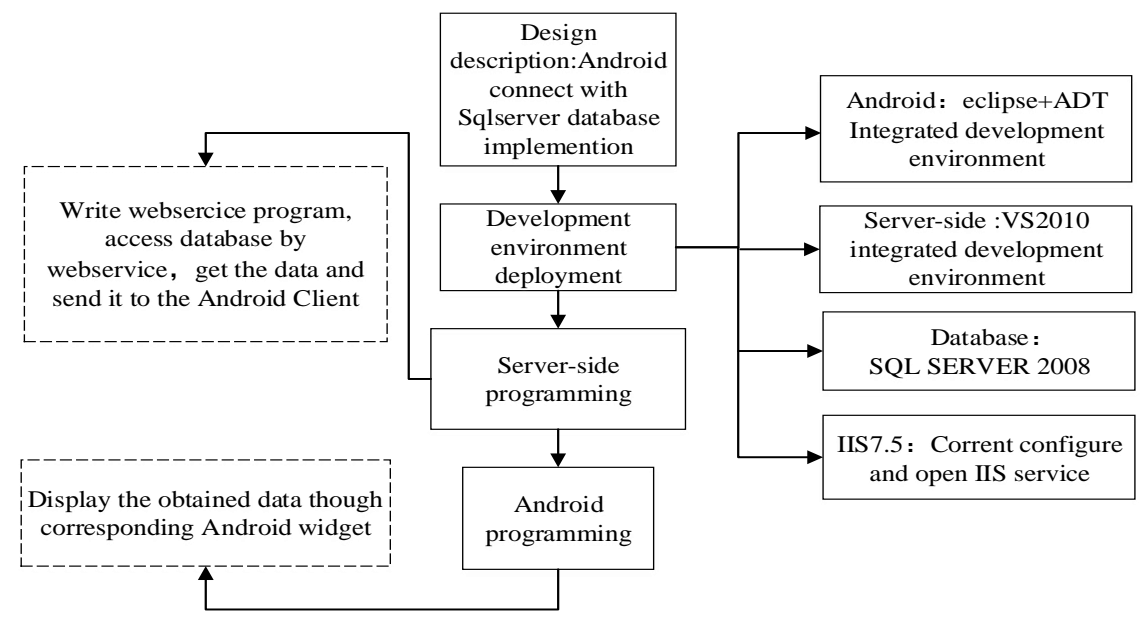

Fig. 7. The Android APP design process

\section{Design of composting monitoring experiments}

The composting monitoring experiments were carried out from January 13 to February 22, 2017, in Linquan Quanyou breeding pig farm, Fuyang city, Anhui Province. Pig manure was taken from this pig farm, with $\mathrm{C} / \mathrm{N}$ : 13: 1 and the moisture content: $70 \%$. The corn stalk was taken from the Zhangguan town of Linquan county, with C/N: 53: 1 and the moisture content: $12 \%$. First, the corn straw was crushed into $2 \mathrm{~cm}$ length, and fresh pig manure was treated by the processing of wet and dry separation. Second, pig manure and corn straw were mixed according to the proportion of 4: 1 and the water content of the pile was $65 \% \sim 75 \%$. Then mixed with inoculum (Bacillus $0.3 \%$ ). Finally, divided into two piles which were named No. 1 compost and No. 2 compost with each weighed about $500 \mathrm{~kg}$. No. 1 composting temperature was collected every ten minutes with the intelligent composting process monitoring device. No. 2 composting temperature was measured at 12:00 noon every day with the mechanical artificial temperature measuring device. Two composting temperature measure devices were inserted in the middle of each compost down at 30 $\mathrm{cm}$. No. 1 compost was turned once every 3 days after the temperature rose to $55^{\circ} \mathrm{C}$. No. 2 compost was turned once every 4 days $[9,10,11]$.

\section{$4 \quad$ Results and Discussion}



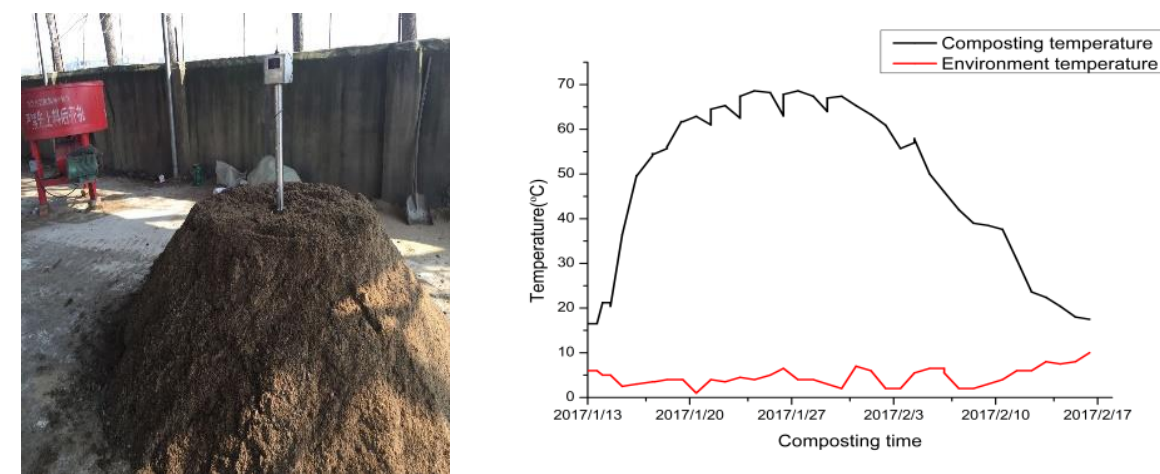

Fig. 8. No. 1 compost and its temperature change
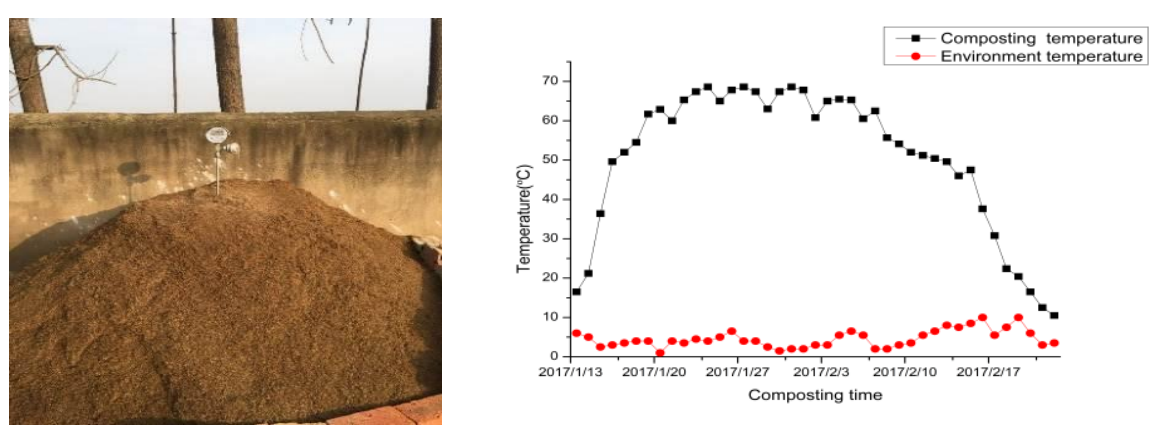

Fig. 9. No. 2 compost and its temperature change

In Figure 8, we could see clearly that the temperature of the compost rose rapidly after composting, and it reached the high temperature degradation stage of composting from fifth days $\left(>50{ }^{\circ} \mathrm{C}\right)$. Then the temperature of $55^{\circ} \mathrm{C}$ or more lasted for 17 days. According to the U.S. Environmental Protection Agency (USEPA) reported the composting temperature was maintained at $55{ }^{\circ} \mathrm{C} 3$ days or $50{ }^{\circ} \mathrm{C}$ for more than 7 days, the pathogenic bacteria could be effectively killed and the sanitary standard of fecal harmless for static aerobic composting could be conformed $[12,13,14]$. After 22 days of fermentation, composting entered the cooling stage. By 34 days, the composting temperature was close to the environment temperature indicating that the compost had been basically rotted [15].

However, in Figure 9, there were about 21 days of the maintenance time at the temperature of $55{ }^{\circ} \mathrm{C}$ or more for No. 2 compost. After 25 days of fermentation, the compost entered the cooling stage, and the composting temperature was not close to the environmental temperature until the fortieth day. From these two sets of composting, it could be seen that the intelligent composting process monitoring device could be used to monitor the whole process of composting. Accordingly, the users could do the dump in a timely manner, which could reduce the composting time. 
In this compost experiments, No. 1 compost took about 6 days less than No. 2 compost.

\section{Conclusions}

In conclusion, an intelligent monitoring system was proposed in this paper, which could achieve the real-time monitoring of the whole process of composting. Users can query the temperature of the compost by web page or smartphone APP with the advantages of high temperature measurement accuracy, simple operation. Accordingly, the users can do the dump in time to improve the inactivation efficiency of pathogenic microorganisms and reduce the loss of compost nutrients. Therefore, this monitoring technology can significantly shorten the composting cycle, improve the compost quality, and have great potential.

Acknowledgments. The authors thank the financial support by the National High Technology Research and Development Program (SS2013AA102302); National Key Research and Development Program (2016YFD0800901-03); National Key Technology Research and Development Program of the Ministry of Science and Technology of China (2014BAD08B11). And thank Linquan Quanyou breeding pig farm (Fuyang city, Anhui Province) for providing experimental conditions.

\section{References}

1. Cesaro, A., Belgiorno, V., Guida. M.: Compost from organic solid waste: Quality assessment and European regulations for its sustainable use. Resources, Conservation and Recycling. 94:72--79(2015)

2. Park, K.: Composting of food waste and mixed poultry manure inoculated with effective microorganisms. Engineering in Agriculture, Environment and Food. 4(4): 106--111(2011)

3. Khelil, O., Cheba, B.: Thermophilic cellulolytic microorganisms from western Algerian Sources: Promising solates for cellulosic biomass recycling. Procedia Technology. 12:519$-528(2014)$

4. Xu, T., Li, L., Zhen, C., Sun, L., Fu, H., Zhang, X.: Effects of different microorganism consortiums on composting process of pig manures and their nutrient status. Transection of the Chinese Society of Agricultural Engineering. 24(11):217--221(2008)

5. Li, H., Li, M., Zhan, K., Yang, X., Weng, S., Yuan, Y., Chen, S., Luo, W., Gao, H.: Intelligent monitoring system for laminated henhouse based on Internet of Things. Transactions of the Chinese Society of Agricultural Engineering. 31(Supp.2):210--215 (2015)

6. Troubleyn, E., Moerman, I., Demeester, P.: QoS challenges in wireless sensor networked robotic. Wireless Personal Communications. 70(3): 1059--1075 (2013)

7. Sharma V., Kumar, R.: A cooperative network framework for multi-UAV guided ground Ad Hoc networks, Journal of Intelligent and Robotic Systems. 77(3/4): 629--652 (2015)

8. Li, Q., Wang, X.C., Zhang, H.H., Shi, H.L., Ngo, H.H.: Characteristics of nitrogen transformation and microbial community in an aerobic composting reactor under two typical temperatures. Bioresource Technology. 137:270--277(2013) 
9. Tandy, S., Healey, J.R., Nason, M.A., Williamson, J.C., Jones, D.L., Thain, S.C.: FT-IR as an alternative method for measuring chemical properties during composting. Bioresource Technology. 101(14):5431--5436(2010)

10. Zhou, J., Wang, L., Xu, Q., Jiang, X.: Optimum ratio of pig manure to edible fungi residue improving quality of organic fertilizer by composting. Transection of the Chinese Society of Agricultural Engineering. 31(7):201-207(2015)

11. Shi, L., Gu, J., Pan, H., Zhang, K., Yin, Y., Zhao, T., Wang, X., Gao, H.: Improving enzyme activity by compound microbial agents in compost with mixed fruit tree branches and pig manure during composting. Transactions of the Chinese Society of Agricultural Engineering. 31(5):244--251(2015)

12. Wang, H., Yang, K., Chen, S., Zhong, H., Wang, Y., Wang, A., Wen, Y., Liu, L., Cai, Z.: Investigation of livestock and poultry manure composting treatment to eliminate parasites and eggs. Journal of Southwest University for Nationalities. 39(03):307--310(2013)

13. China National Standardization Management Committee: Sanitary standard for the nonhazardous treatment of night soil (GB7959-2012). Standards Press of China. Beijing (2013)

14. Cao, Y., Chang, Z., Huang, H., Xu, Y., Li, C., Wu, H.: Effect of compost inoculation on pig manure composting. Transactions of the Chinese Society of Agricultural Engineering. 31(21):220--226(2015)

15. Huang, X., Han, Z., Shi, D., Huang, X., Wu, W., Liu, Y.: Nitrogen loss and its control during livestock manure composting. Chinese Journal of Applied Ecology. 21(1):247-254(2010) 